\title{
Comments on a Massless Neutralino
}

\author{
H. K. Dreiner \\ Bethe Center for Theoretical Physics \& Physics Institute, University of Bonn, Bonn, Germany
}

\begin{abstract}
We consider first an interesting connection between the development of physics and the Boston Red Sox. We then discuss in detail the collider phenomenology, as well as precision electroweak observables of a very light neutralino. We conclude by considering also the astrophysics and cosmology of a very light neutralino. We find that a massless neutralino is consistent with all present data.
\end{abstract}

Keywords: Neutralinos, e+e- collider, electroweak precision observables, supernovae, dark matter PACS: $12.60 . J v, 13.66 . \mathrm{Hk}, 13.66 . \mathrm{Jn}, 14.80 . \mathrm{Nb}, 97.60 . \mathrm{Bw}, 95.35 .+\mathrm{d}$

\section{PREFACE}

It is a pleasure to speak here at Northeastern University, in Boston. I was born not too far, in Williamstown, some time ago, but have lived in Europe for quite some time. Now, it is great to be back in Massachusetts. There is of course a longstanding connection between scientific circles in Europe and this wonderful city and state. It is even rumored that Galileo Galilei (1564-1642) was offered a job next door at Harvard (est. 1635). However, Galileo Galilei never came, possibly because he wasn't sure of getting tenure, or possibly because of earlier support for Boston from fellow Italian Leonardo Davinci (1452-1519), see Fig. 11a. Galieli always did want to be different. This strong affiliation to Boston has been maintained through the years by Nicolaus Copernicus (1473-1543), Fig. 1b, and Johannes Kepler (1571-1630) who worked in Tübingen and Prag but dreamt of Red Sox nation, Fig. 1k. In more modern times Lise Meitner Fig. 1d and Werner Heisenberg, Fig. $2 \mathrm{a}$, have upheld the torch. In the $20^{\text {th }}$ century, the culmination of this connection in the person of Albert Einstein, Fig. 2 b, is well documented.

1903 Red Sox win $1^{\text {st }}$ ever World Series on grounds of Northeastern

1904 Red Sox win AL pennant3

1905 - Einstein, inspired, has the season of his life

'10's Red Sox dominant force

1916 —- Einstein: General Relativity

1918 Sox win last World Series for a while

1919 Babe Ruth Sold

1919 —- Einstein, past his prime, slumps

2004 Red Sox win Series again, hurrah!

2005 World celebrates Einstein centennial, double hurrah!

$2005+-$ Both Red Sox and Einstein in $7^{\text {th }}$ heaven 


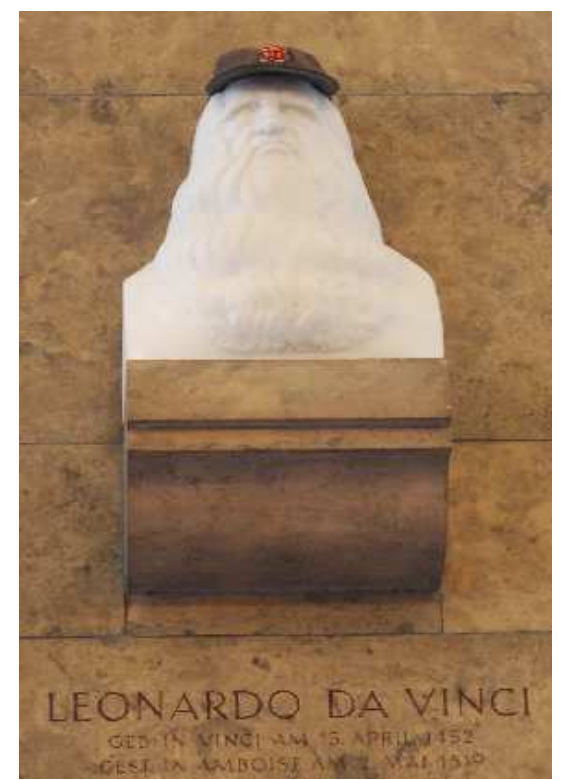

(a)

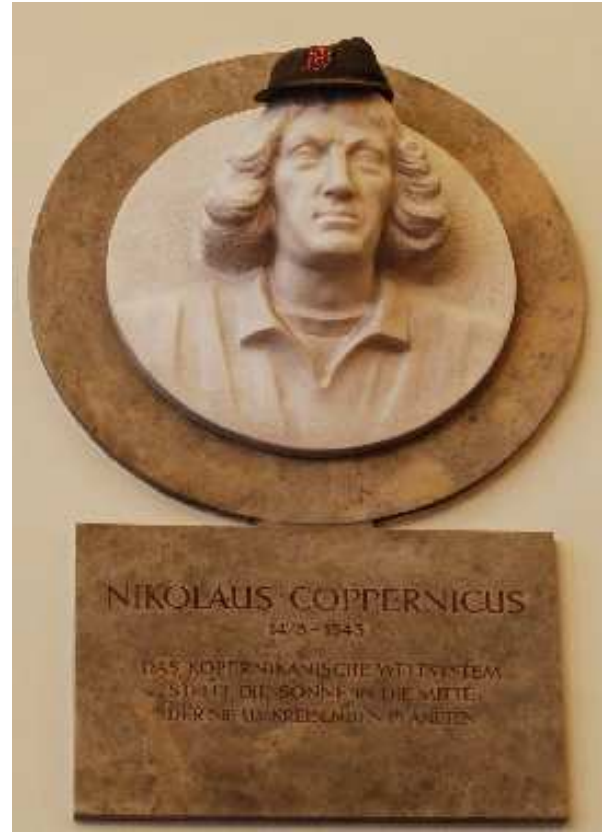

(b)

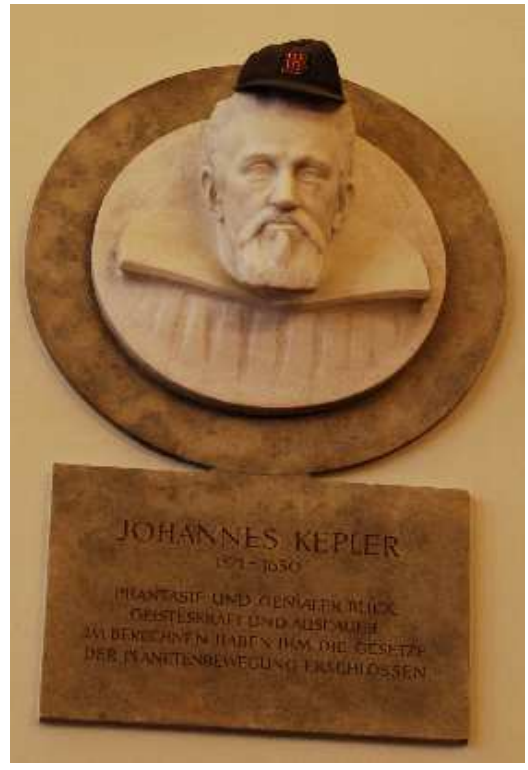

(c)

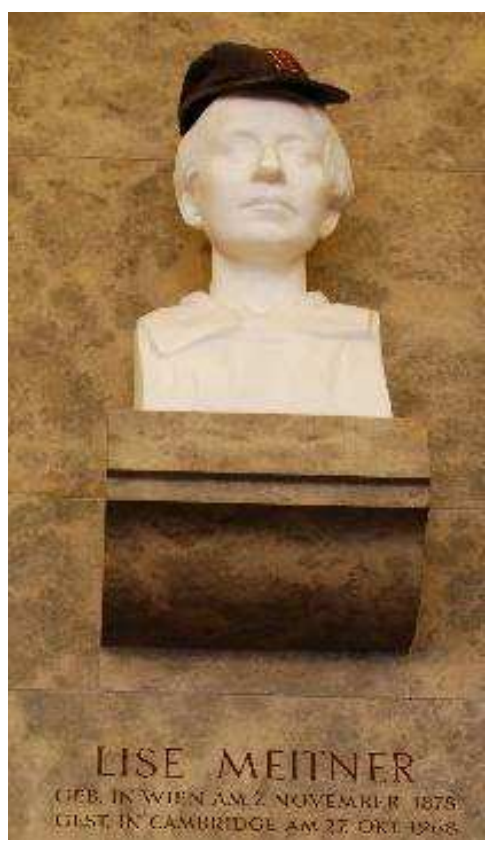

(d)

FIGURE 1. (a) Leonardo Davinci, (b) Nikolaus Copernicus (c) Johannes Kepler, and (d) Lise Meitner showing their support.

\section{INTRODUCTION}

The lightest supersymmetric particle, the LSP, plays a special role in the search for supersymmetry at colliders. It is the end product of the cascade decay of any produced 

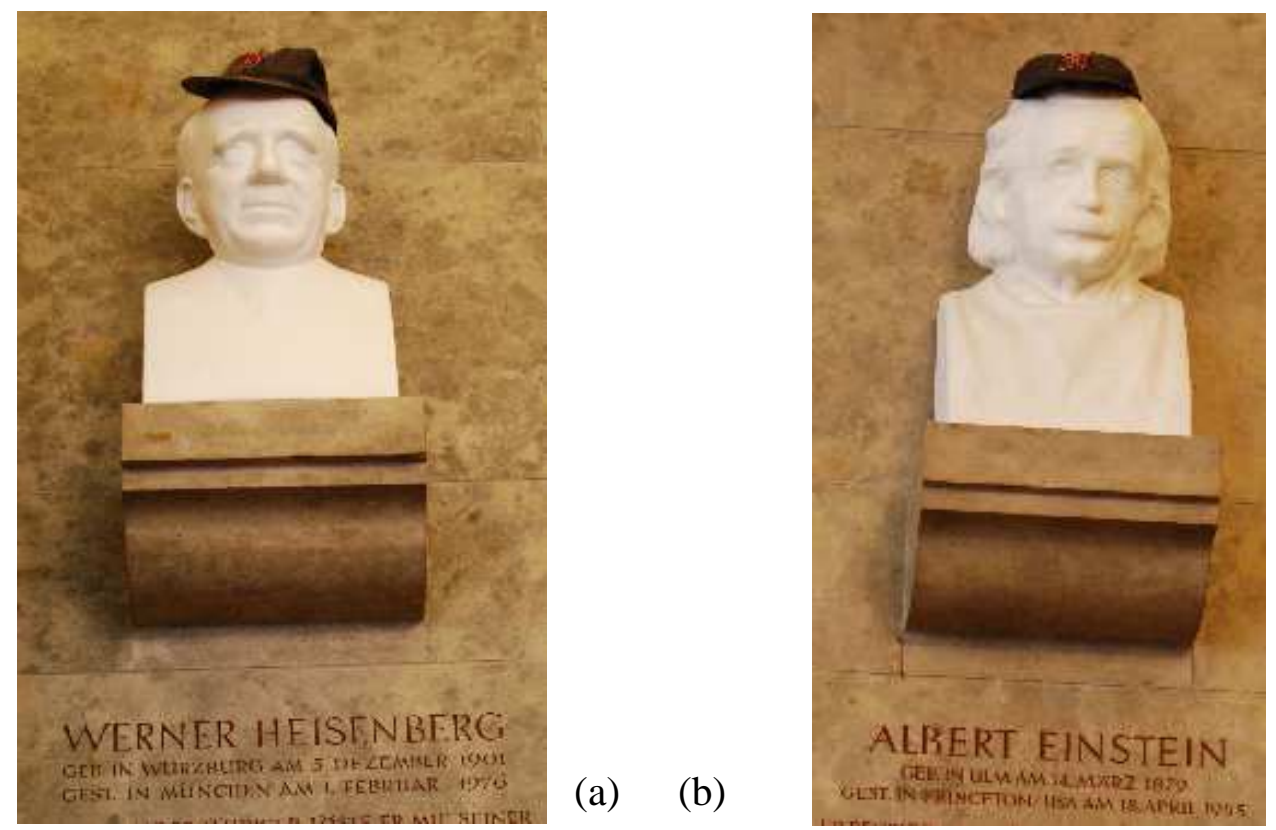

FIGURE 2. Also (a) Werner Heisenberg and (b) Albert Einstein are dedicated followers.

SUSY particle. Thus the nature of the LSP is decisive for all supersymmetric signatures at the LHC. For conserved proton hexality [1, 2] the LSP is stable and must be the lightest neutralino: $\tilde{\chi}_{1}^{0}$. Here enquire: 'How light can the $\tilde{\chi}_{1}^{0}$ be?'. This is a summary of previous work [3, 4, 5, 6, 7, 8]. The PDG cites as the laboratory bound [9]

$$
m_{\tilde{\chi}_{1}^{0}}>46 \mathrm{GeV}
$$

at $95 \%$ C.L., which is based on the chargino searches at LEP. These yield lower limits on $M_{2}$ and $\mu$. Furthermore, this bound assumes an underlying SUSY GUT, i.e. $M_{1}=$ $\frac{5}{3} \tan ^{2} \theta_{\mathrm{w}} M_{2} \approx \frac{1}{2} M_{2}$. The experimental bound on $M_{2}$ then implies a lower bound on $M_{1}$. Using the neutralino mass matrix, together these give rise to the lower bound in Eq. (1).

It is the purpose of this paper to investigate the consequences of dropping the SUSY GUT assumption. In this more general scenario, $M_{1}$ and $M_{2}$ are both free parameters. We systematically demonstrate that then a massless neutralino is consistent with theory and all present laboratory data. For possible models see [10, 11] and techniques [12]. Taking the determinant of the neutralino mass matrix [8] and setting it to zero, we get

$$
\mu\left[M_{2} M_{Z}^{2} s_{\mathrm{w}}^{2} \sin (2 \beta)+M_{1}\left(-M_{2} \mu+M_{Z}^{2} c_{\mathrm{w}}^{2} \sin (2 \beta)\right)\right]=0 .
$$

The solution $\mu=0$ is excluded by the LEP chargino bounds. Solving for $M_{1}$ yields

$$
M_{1}=\frac{M_{2} M_{Z}^{2} \sin (2 \beta) s_{\mathrm{w}}^{2}}{\mu M_{2}-M_{Z}^{2} \sin (2 \beta) c_{\mathrm{w}}^{2}} .
$$


Thus for every value of $M_{2}, \mu$ and $\tan \beta$, we can find a value of $M_{1}$ with $M_{\tilde{\chi}_{1}^{0}}=0$. This is stable under radiative corrections [8]. In the case of complex gaugino parameters there is not always a solution. In the real case, Eq. (3) leads to $M_{1} \approx M_{2} / 40$. We find that for very light neutralinos they are typically more than $90 \%$ bino.

\section{COLLIDER BOUNDS}

Neutralino production at LEP: If we assume $m_{\tilde{\chi}_{1}^{0}}=0$ the associated production, $e^{+} e^{-} \rightarrow \tilde{\chi}_{1}^{0} \tilde{\chi}_{2}^{0}$, would be accessible at LEP up to the kinematical limit of $\sqrt{s}=m_{\tilde{\chi}_{2}^{0}}=$ $208 \mathrm{GeV}$. In order to compare with the results of the LEP searches we make use of the model-independent upper bounds on the topological neutralino production cross section obtained by OPAL with $\sqrt{s}=208 \mathrm{GeV}$ [13],

$$
\sigma\left(e^{+} e^{-} \rightarrow \tilde{\chi}_{1}^{0} \tilde{\chi}_{2}^{0}\right) \times \mathrm{BR}\left(\tilde{\chi}_{2}^{0} \rightarrow Z \tilde{\chi}_{1}^{0}\right) \times \mathrm{BR}(Z \rightarrow q \bar{q})
$$

Taking into account $\mathrm{BR}(Z \rightarrow q \bar{q}) \approx 70 \%$, one can read off from the OPAL plots [13],

$$
\sigma\left(e^{+} e^{-} \rightarrow \tilde{\chi}_{1}^{0} \tilde{\chi}_{2}^{0}\right) \times \mathrm{BR}\left(\tilde{\chi}_{2}^{0} \rightarrow Z \tilde{\chi}_{1}^{0}\right)<70 \mathrm{fb} .
$$

We analyze this bound assuming conservatively that $\operatorname{BR}\left(\tilde{\chi}_{2}^{0} \rightarrow Z \tilde{\chi}_{1}^{0}\right)=1$. Imposing the bound, Eq. (5), significantly constrains the parameter space. In Fig. 3 (a) we show contour lines of the cross section $\sigma\left(e^{+} e^{-} \rightarrow \tilde{\chi}_{1}^{0} \tilde{\chi}_{2}^{0}\right)$ in the $\mu-M_{2}$ plane for $\tan \beta=10$ and degenerate selectron masses $m_{\tilde{e}_{R}}=m_{\tilde{e}_{L}}=m_{\tilde{e}}=200 \mathrm{GeV}$. We observe that there is a large region where $\sigma>70 \mathrm{fb}$. Here the selectron masses have to be sufficiently heavy. Thus, the bound on the neutralino production cross section can be translated into lower bounds on the selectron mass $m_{\tilde{e}}$, for $m_{\tilde{\chi}_{1}^{0}}=0$. In Fig. 3 (b), we show contours of the selectron mass, such that the bound $\sigma\left(e^{+} e^{-} \rightarrow \tilde{\chi}_{1}^{0} \tilde{\chi}_{2}^{0}\right)<70 \mathrm{fb}$ is fulfilled.

Radiative neutralino production: An additional search channel is radiative neutralino production, $e^{+} e^{-} \rightarrow \tilde{\chi}_{1}^{0} \tilde{\chi}_{1}^{0} \gamma$. Due to the large SM background, $e^{+} e^{-} \rightarrow v \bar{v} \gamma$, the significance is at best $S \approx 0.1$ for $\mathscr{L}=100 \mathrm{pb}^{-1}$ and $\sqrt{s}=208 \mathrm{GeV}$ [5, 6]. Cuts on the photon energy or angle do not help, due to similar distributions of signal and background. We find a similar situation at $b$-factories, $\sqrt{s} \approx 10 \mathrm{GeV}$. An identification of the signal 'photon plus missing energy' is difficult due to the large photonic background from the abundant hadronic processes. At the ILC, radiative neutralino production would be measurable, due to the option of polarised beams [3, 5, 6, 14].

\section{PRECISION OBSERVABLES}

In the following we study the impact of a light or massless neutralino on electroweak precision physics. We consider the full one-loop and leading higher-order corrections $[15,16,17,18]$. We focus on the invisible $Z^{0}$ width, $\Gamma_{\text {inv }}$, as an example. The additional contributions due to $\Gamma_{\tilde{\chi}_{1}^{0}}=\Gamma\left(Z \rightarrow \tilde{\chi}_{1}^{0} \tilde{\chi}_{1}^{0}\right)$ can be large if the neutralino has a considerable Higgsino component. In Ref. [19], the processes $Z \rightarrow \tilde{\chi}_{1}^{0} \tilde{\chi}_{1}^{0}$ and $Z \rightarrow f \bar{f}$ have been 

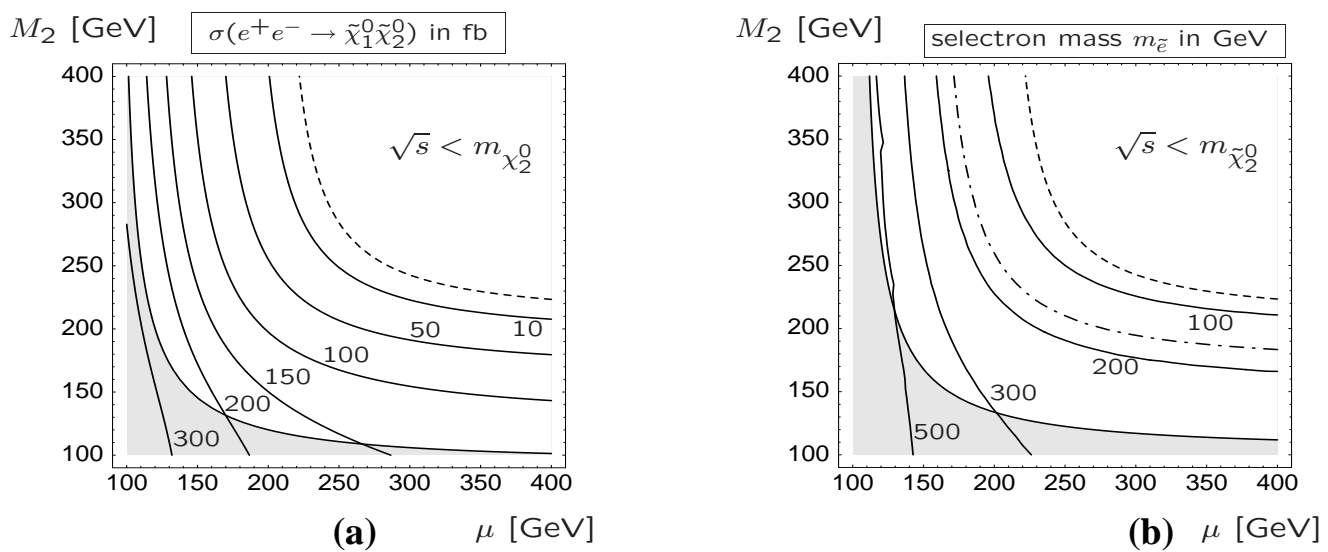

FIGURE 3. (a) Contour lines of $\sigma\left(e^{+} e^{-} \rightarrow \tilde{\chi}_{1}^{0} \tilde{\chi}_{2}^{0}\right)$ with $\tan \beta=10$, and $m_{\tilde{e}}=200 \mathrm{GeV}$, at $\sqrt{s}=208 \mathrm{GeV}$. (b) Lower bounds on the selectron mass $m_{\tilde{e}}$, such that $\sigma\left(e^{+} e^{-} \rightarrow \tilde{\chi}_{1}^{0} \tilde{\chi}_{2}^{0}\right)=70 \mathrm{fb}$.

calculated at $\mathscr{O}(\alpha)$ and supplemented with leading higher-order terms from the SM and the MSSM. The experimental values for the total width and the invisible width of the $Z$ boson are [9]

$$
\Gamma_{Z}^{\exp }=2495.2 \pm 2.3 \mathrm{MeV}, \quad \Gamma_{\text {inv }}^{\exp }=499.0 \pm 1.5 \mathrm{MeV} .
$$

In our numerical analysis, we show the results for

$$
\delta \Gamma_{\text {inv }} \equiv \Gamma_{\text {inv }}-\Gamma_{\text {inv }}^{\exp },
$$

We investigate $\delta \Gamma_{\text {inv }}$ in one representative SUSY parameter regions. We choose fairly light scalar fermions and set the diagonal soft SUSY-breaking parameter $m_{\tilde{f}}$ to $250 \mathrm{GeV}$. In Fig. 4, we show $\delta \Gamma_{\text {inv }}$ as a function of $M_{2}$ and $\mu . M_{1}$ is fixed via Eq. (3). The remaining SUSY parameters are $\tan \beta=10, A_{\tau}=A_{t}=A_{b}=m_{\tilde{g}}=M_{A}=500 \mathrm{GeV}$. Here $A_{f}$ denotes the trilinear couplings of the Higgses to the sfermions, $m_{\tilde{g}}$ is the gluino mass, and $M_{A}$ the mass of the CP-odd Higgs boson. The deviations from the experimental central values as given in Eqs. (7), are indicated as experimental $n \times \sigma$ contours of the respective observable. In addition, the 95\% C.L. exclusion bounds of $m_{\tilde{\chi}_{1}^{ \pm}}>94 \mathrm{GeV}$ [9] on the chargino mass from direct searches are marked by dashed white lines. Fig. 4 clearly displays that for both observables the MSSM prediction can deviate considerably from the experimental values. This is in particular the case for small $|\mu|$ and small $\left|M_{2}\right|$. Nearly all of the parameter space ruled out at the $5 \sigma$ level for $\Gamma_{\text {inv }}$ is, however, already excluded due to direct chargino searches. For the interpretation of these plots it is furthermore important to keep in mind that the results for $\Gamma_{\mathrm{inv}}$ do not only depend on $\mu$ and $M_{2}$, but on all the other SUSY parameters as well. This means in particular that an apparent $1 \sigma$ effect can easily be caused or canceled out by, for instance, a change induced by $m_{\tilde{f}}$, which is known to have a strong impact on the decay into SM fermions, see also the discussion in Ref. [19]. Furthermore even in the SM, $\Gamma_{\text {inv }}$ is predicted to be 


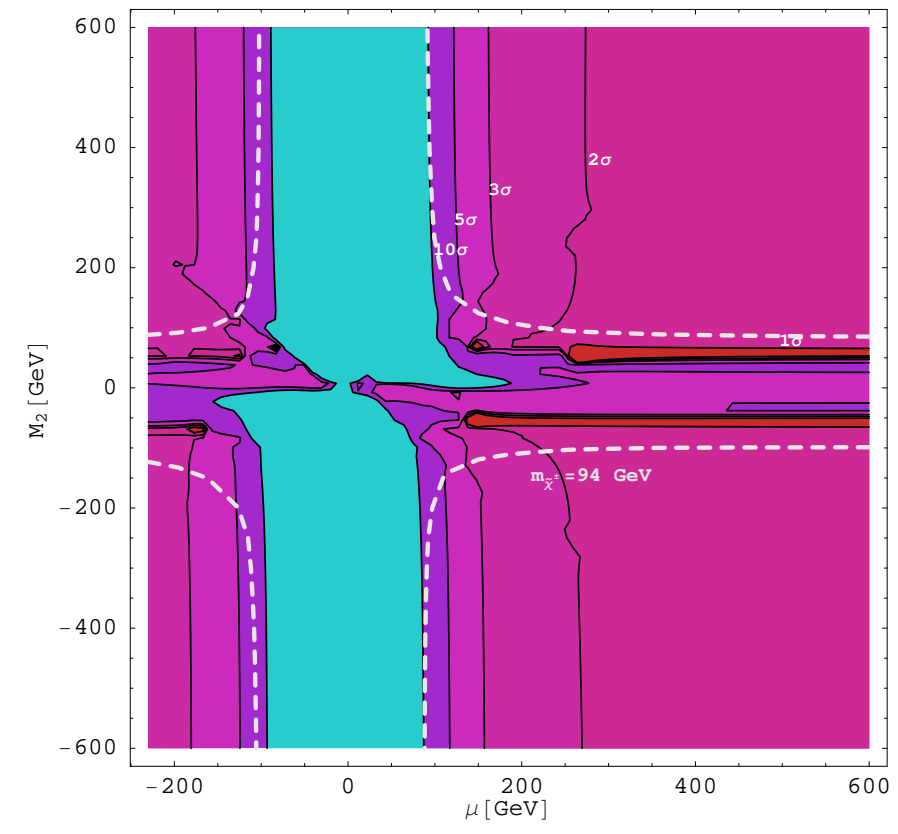

FIGURE 4. The difference of the experimental value and the theory prediction for the invisible $Z$ width, $\delta \Gamma_{\text {inv }}$, indicated as $\delta \Gamma_{\text {inv }} \equiv\left(\Gamma_{\text {inv }}-\Gamma_{\text {inv }}^{\exp }\right)=(10,5,3,2,1) \times \sigma_{\Gamma_{\text {inv }}}^{\exp }$ contours.

slightly larger than the experimentally measured value, resulting in a $\sim 1 \sigma$ deviation. In summary, $\Gamma_{\text {inv }}$ cannot exclude a massless neutralino. The parts of the $\mu-M_{2}$ planes that lead to a large deviation from the experimental values are mostly already excluded by direct chargino searches.

\section{ELECTRIC DIPOLE MOMENTS AND RARE MESON DECAYS}

For the electric dipole moments we refer the reader to the original paper [8]. For the rare meson decays we defer to the dedicated talk by Ben O'Leary (RWTH Aachen) also given at this conference [20, 21]. For some relevant techniques see [22, 23]

\section{ASTROPHYSICS AND COSMOLOGY}

Supernova Cooling: A very light neutralino, $M_{\chi} \leq \mathscr{O}(100 \mathrm{MeV})$ can contribute to supernova cooling [4, 24, 25]. The two main production mechanisms are

$$
e^{+}+e^{-} \longrightarrow \tilde{\chi}_{1}^{0}+\tilde{\chi}_{1}^{0}, \quad N+N \longrightarrow N+N+\tilde{\chi}_{1}^{0}+\tilde{\chi}_{1}^{0} .
$$

Once produced, the neutralinos have a mean-free-path, $\lambda_{\tilde{\chi}_{1}^{0}}$, in the supernova core which is determined via the cross sections for the processes

$$
\tilde{\chi}_{1}^{0}+e \longrightarrow \tilde{\chi}_{1}^{0}+e, \quad \tilde{\chi}_{1}^{0}+N \longrightarrow \tilde{\chi}_{1}^{0}+N,
$$




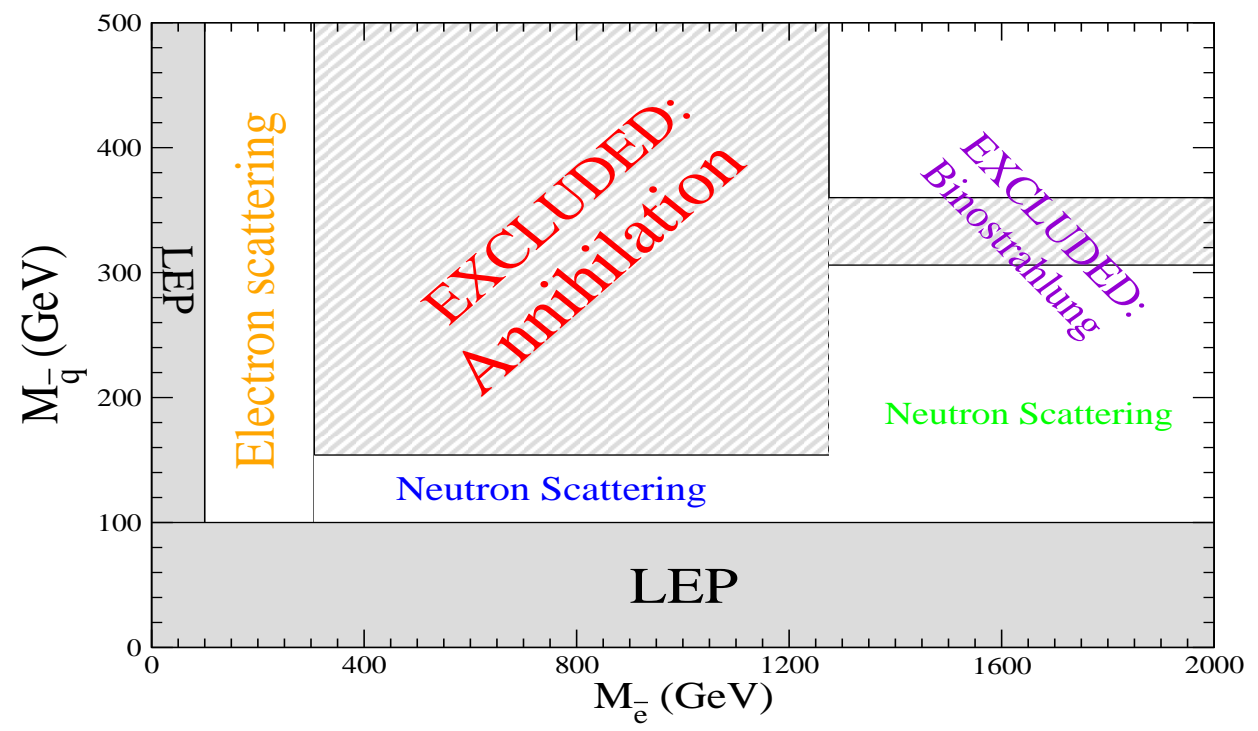

FIGURE 5. Exclusion in SUSY plane for a massless neutralino from supernova cooling [4].

In order to retain the observed neutrino signal we obtain the exclusion regions depicted in Fig. 5, These depend strongly on the selectron and squark masses which enter in the propagators of the processes Eqs. (8), (9).

Hot Dark Matter, the Cowsik-McClelland Bound: Here, we consider the case of a (nearly) massless neutralino, $m_{\tilde{\chi}_{1}^{0}} \lesssim \mathscr{O}(1 \mathrm{eV})$. Since the very light bino contributes to the hot dark matter of the universe, we assume here implicitly that the cold dark matter originates from another source. The bino relic energy density, $\rho_{\widetilde{\mathscr{B}}}$, divided by the critical energy density of the universe, $\rho_{c}$, is given by [26]

$$
\Omega_{\tilde{\mathscr{B}}} \equiv \frac{\rho_{\widetilde{\mathscr{B}}}}{\rho_{c}}=\frac{43}{11} \zeta(3) \frac{8 \pi G_{N}}{3 H_{0}^{2}} \frac{g_{\mathrm{eff}}(\widetilde{\mathscr{B}})}{g_{* S}(T)} T_{\gamma}^{3} m_{\widetilde{\mathscr{B}}} .
$$

In order for the bino hot dark matter not to disturb the structure formation, we assume its contribution to be less than the upper bound on the energy density of the neutrinos, as determined by the WMAP data [27]

$$
\Omega_{\widetilde{\mathscr{B}}} h^{2} \leq\left[\Omega_{v} h^{2}\right]_{\max }=0.0076 .
$$

From Eqs. (10) and (11), we find the conservative upper bound

$$
m_{\widetilde{B}} \leq 0.07 \mathrm{eV} \text {. }
$$

Thus a very light bino with mass below about $0.1 \mathrm{GeV}$ is consistent with structure formation. This line of argument was originally used by Gershtein and Zel'dovich [28] and Cowsik and McClelland [29] to derive a neutrino upper mass bound, by requiring $\Omega_{v} \leq 1$. We have here obtained an upper mass bound for a hot dark matter bino. 
Acknowledgments I would like to thank Christoph Hanhart, Sven Heinemeyer, Olaf Kittel, Ulrich Langenfeld, Daniel Phillips and Georg Weiglein for the collaborations underlying this work. This work was partially supported by SFB TR-33 The Dark Universe. I thank the Deutsche Museum, München, for hospitality during a "Physikshow Bonn" visit, in March 2009.

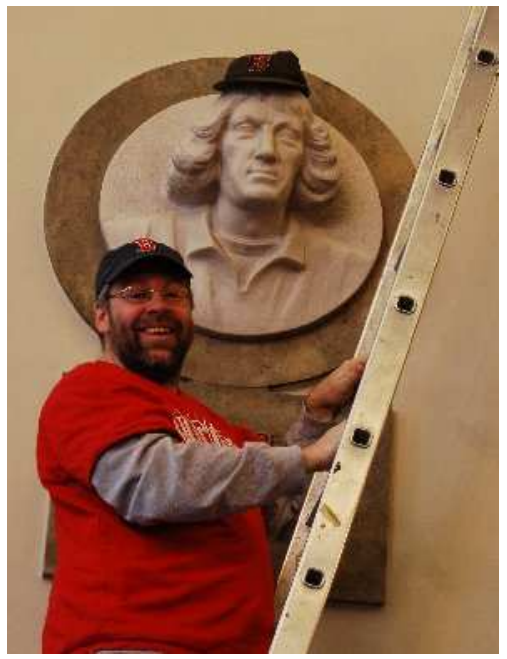

REFERENCES

1. H. Dreiner, C. Luhn and M. Thormeier, Phys. Rev. D 73 (2006) 075007 [arXiv:hep-ph/0512163].

2. H. Dreiner, et al. Nucl. Phys. B 795 (2008) 172 [arXiv:0708.0989 [hep-ph]].

3. D. Choudhury, et al. Phys. Rev. D 61 (2000) 095009 [arXiv:hep-ph/9911365]; A. Dedes, H. Dreiner, P. Richardson, Phys. Rev. D 65 (2002) 015001 |arXiv:hep-ph/0106199|.

4. H. Dreiner, et al., Phys. Rev. D 68 (2003) 055004 [arXiv:hep-ph/0304289].

5. H. Dreiner, O. Kittel and U. Langenfeld, Phys. Rev. D 74 (2006) 115010 [arXiv:hep-ph/0610020].

6. H. Dreiner, O. Kittel and U. Langenfeld, Eur. Phys. J. C 54 (2008) 277 [arXiv:hep-ph/0703009].

7. H. Dreiner, et al. arXiv:0707.1425 [hep-ph].

8. H. K. Dreiner, et al., Eur. Phys. J. C 62 (2009) 547 [arXiv:0901.3485 [hep-ph]].

9. C. Amsler et al. [Particle Data Group], Phys. Lett. B 667 (2008) 1.

10. A. H. Chamseddine and H. K. Dreiner, Nucl. Phys. B 458 (1996) 65 [arXiv:hep-ph/9504337].

11. E. Dudas, S. Lavignac and J. Parmentier, Nucl. Phys. B 808 (2009) 237 [arXiv:0808.0562 [hep-ph]].

12. H. Dreiner, H. Haber and S. Martin, arXiv:0812.1594 [hep-ph].

13. G. Abbiendi et al. [OPAL Collaboration], Eur. Phys. J. C 35 (2004) 1 [arXiv:hep-ex/0401026].

14. G. Moortgat-Pick et al. Phys. Rept. 460 (2008) 131 [arXiv:hep-ph/0507011].

15. A. Djouadi, et al. Phys. Rev. Lett. 78 (1997) 3626; Phys. Rev. D 57 (1998) 4179 [hep-ph/9710438].

16. S. Heinemeyer, G. Weiglein, JHEP 0210 (2002) 072 [hep-ph/0209305]; hep-ph/0301062

17. J. Haestier, et al., JHEP 0512 (2005) 027 [arXiv:hep-ph/0508139]; hep-ph/0506259

18. S. Heinemeyer, et al. JHEP 0608, 052 (2006) [arXiv:hep-ph/0604147].

19. S. Heinemeyer, et al. JHEP 0804 (2008) 039 [arXiv:0710.2972 [hep-ph]].

20. B. O'Leary, arXiv:0909.3994 [hep-ph].

21. H. K. Dreiner, et al, Phys. Rev. D 80, 035018 (2009) [arXiv:0905.2051 [hep-ph]].

22. H. K. Dreiner, G. Polesello, M. Thormeier, Phys. Rev. D 65, 115006 (2002) [arXiv:hep-ph/0112228].

23. H. K. Dreiner, M. Kramer and B. O'Leary, Phys. Rev. D 75, 114016 (2007) [arXiv:hep-ph/0612278].

24. J. Grifols, E. Masso and S. Peris, Phys. Lett. B 220 (1989) 591.

25. J. Ellis, K. Olive, S. Sarkar and D. Sciama, Phys. Lett. B 215, 404 (1988).

26. E. Kolb and M. Turner "The Early Universe" (Westview Press 1990).

27. J. Dunkley et al. [WMAP Collaboration], arXiv:0803.0586 [astro-ph].

28. S. Gershtein and Y. Zeldovich, JETP Lett. 4 (1966) 120 [Pisma Zh. Eksp. Teor. Fiz. 4 (1966) 174].

29. R. Cowsik and J. McClelland, Phys. Rev. Lett. 29 (1972) 669. 